\title{
Latest astronomical constraints on some nonlinear parametric dark energy models
}

\author{
Weiqiang Yang,, , S Supriya Pan, ${ }^{2,3, \dagger}$ and Andronikos Paliathanasis ${ }^{4,5}$, 用 \\ ${ }^{1}$ Department of Physics, Liaoning Normal University, Dalian, 116029, P. R. China \\ ${ }^{2}$ Department of Physical Sciences, Indian Institute of Science Education and Research, \\ Kolkata, Mohanpur-741246, West Bengal, India \\ ${ }^{3}$ Department of Mathematics, Raiganj Surendranath Mahavidyalaya, \\ Sudarshanpur, Raiganj, West Bengal 733134, India \\ ${ }^{4}$ Instituto de Ciencias Fúsicas y Matemáticas, Universidad Austral de Chile, 5090000 Valdivia, Chile \\ ${ }^{5}$ Institute of Systems Science, Durban University of Technology, \\ PO Box 1334, Durban 4000, Republic of South Africa
}

\begin{abstract}
We consider nonlinear redshift-dependent equation of state parameters as dark energy models in a spatially flat Friedmann-Lemaître-Robertson-Walker universe. To depict the expansion history of the universe in such cosmological scenarios, we take into account the large scale behaviour of such parametric models and fit them using a set of latest observational data with distinct origin that includes cosmic microwave background radiation, Supernove Type Ia, baryon acoustic oscillations, redshift space distortion, weak gravitational lensing, Hubble parameter measurements from cosmic chronometers and finally the local Hubble constant from Hubble space telescope. The fitting technique avails the publicly available code Cosmological Monte Carlo (CosmoMC), to extract the cosmological information out of these parametric dark energy models. From our analysis it follows that those models could describe the late time accelerating phase of the universe, while they are distinguished from the $\Lambda$-cosmology.
\end{abstract}

PACS numbers: 98.80.-k, 95.36.+x, 95.35.+d, 98.80.Es

\section{INTRODUCTION}

Astronomical observations from various independent sources consistently suggest that our Universe is expanding in an accelerating manner [1 6 . Various approaches have been proposed in the literature. Those apporaches can be classified into two big categories. The dark energy models in which an exotic fluid is introduced in Einstein grvaity, and the modified theories of gravity in which geometrodynamical quantities are introduced in the gravitational action to explain the accelerating phase of the universe. Of course these two categories include in common the cosmological constant. Because the latter can be seen either as a perfect fluid with negative equation of state parameter or as a modification of the EinsteinHilbert Action.

Here we are interested on the parametric dark energy models. In particular, we consider the existence of an exotic dark energy fluid with time-varying equation of state parameter in order to perform the fittings of the cosmological observations. The theoretical origin of those parametric equation of state parameters can be from the dark energy models [7-15], or from the modified theories of gravity [16] 25] (see also the references therein).

In the last decades different parametrizations for the dark energy equation of state have been proposed in the literature which include some well known parametrizations, namely, linear parametrization [26 28], Chevallier-

\footnotetext{
*Electronic address: d11102004@163.com

${ }^{\dagger}$ Electronic address: span@research.jdvu.ac.in

$\ddagger$ Electronic address: anpaliat@phys.uoa.gr
}

Polarski-Linder (known as CPL) parametrization 29, 30], logarithmic parametrization [31, Jassal-BaglaPadmanabhan (known as JBP) parametrization [32, Barboza-Alcaniz parametrization [33, and many more, see also [34 39] and the references therein.

In this work we consider that the fluids of the dark energy sector are non-interacting while for the underlying geometry we consider the spatially flat FriedmannLemaître-Robertson-Walker (FLRW) spacetime. Moreover, we assume that the total energy density of the universe is shared by baryons, radiation, dark matter and dark energy. The dark matter is considered to be pressureless while the dark energy fluid is barotropic and represented by some parametric models. To check the robustness of those parametric dark energy models we consider their large scale behaviour and fit them using the latest observational data from different astronomical probes, namely, cosmic microwave background radiation [40, 41, Supernove Type Ia sample [42, baryon acoustic oscillation distance measurements 43, 45, redshift space distortion [45], weak gravitational lensing [46, 47] and finally the history of the Hubble parameter that combines the latest complilation of the cosmic chronometers 48 and the local Hubble constant 49. From our analysis we see that the parametric dark energy models could be an effective way to study the late time accelerating phase of the universe. The observational constraints on the models suggest that the current value of the dark energy equation of state is close to that of the cosmological constant but the models are distinguished between them and from the cosmological constant as well.

We discuss the background cosmology and introduce the parameteric dark energy models in section 2. The 
perturbation equations for the dark energy models have been written in section 3 . The observational data used in our analysis have been summarized in section 4 and consequenlty the section 5 contains the results of our statistical analysis. Finally, we close our work in section 6 with a brief outline of the whole work.

\section{PARAMETRIC DARK ENERGY MODELS}

The cosmological scenario of our consideration is that of a four dimensional spacetime which is isotropic, homogeneous and spatially flat. In particular the line element, i.e. the metric, is invariant under the action of the Euclidean group $E^{3}$. The corresponding line element of the FLRW spacetime is,

$$
d s^{2}=-N^{2}(t) d t^{2}+a(t)^{2}\left(d r^{2}+r^{2}\left(d \theta^{2}+\sin ^{2} \theta d \phi^{2}\right)\right),
$$

where $a(t)$ is the scalar factor of the universe and $N(t)$ is the lapse function which without any loss of generality we can consider that $N(t)=1$. Furthremore, we consider the comoving observer $u^{a}=\frac{1}{N(t)} \delta_{t}^{a}$, such that $u^{a} u_{a}=$ -1 .

For such spacetime the Einstein's field equation are calculated as

$$
\begin{gathered}
H^{2}=\frac{8 \pi G}{3}\left(\rho_{r}+\rho_{b}+\rho_{c}+\rho_{x}\right), \\
2 \dot{H}+3 H^{2}=-8 \pi G\left(p_{r}+p_{b}+p_{c}+p_{x}\right),
\end{gathered}
$$

where $H=\dot{a} / a$, is the Hubble rate of this universe; $\left(\rho_{i}, p_{i}\right)$ stand respectively, for the energy density and the pressure of the $i$-th fluid where $i$ runs over radiation $(r)$, baryons $(b)$, dark matter $(c)$ and a dark energy fluid $(x)$.

Furthermore, we consider that all the fluid components are minimally coupled to gravity and there is no interaction between the fluids. Hence, the Bianchi identity leads to $\nabla_{\nu} T^{\mu \nu}=0$, in which the radiation and baryons follow the evolution laws $\rho_{r} \propto\left(a / a_{0}\right)^{-4}, \rho_{b} \propto\left(a / a_{0}\right)^{-3}$, respectively, while for the other two components, we have

$$
\rho_{c, x}=\rho_{c, x}^{0}\left(\frac{a}{a_{0}}\right)^{-3} \exp \left(-3 \int_{a_{0}}^{a} \frac{w_{c, x}(\sigma)}{\sigma} d \sigma\right) .
$$

This is a general evolution equation for both dark matter and dark energy with equations of state $w_{c}, w_{x}$, respectively where $\rho_{c, x}^{0}$ is a shorthand notation that describes both $\rho_{c}^{0}$ and $\rho_{x}^{0}$ as the present values of the corresponding energy densities, $a_{0}$ is the present value of the scale factor which is related to the cosmological redshift $z$, as $a_{0} a^{-1}=1+z$. For constant $w_{c}$, the above equation becomes $\rho_{c}=\rho_{c}^{0}\left(a / a_{0}\right)^{-3\left(1+w_{c}\right)}$. In this work, we shall consider pressureless dark matter, that is $w_{c}=0$, but the equation of state for DE is dynamical (time-varying, i.e.

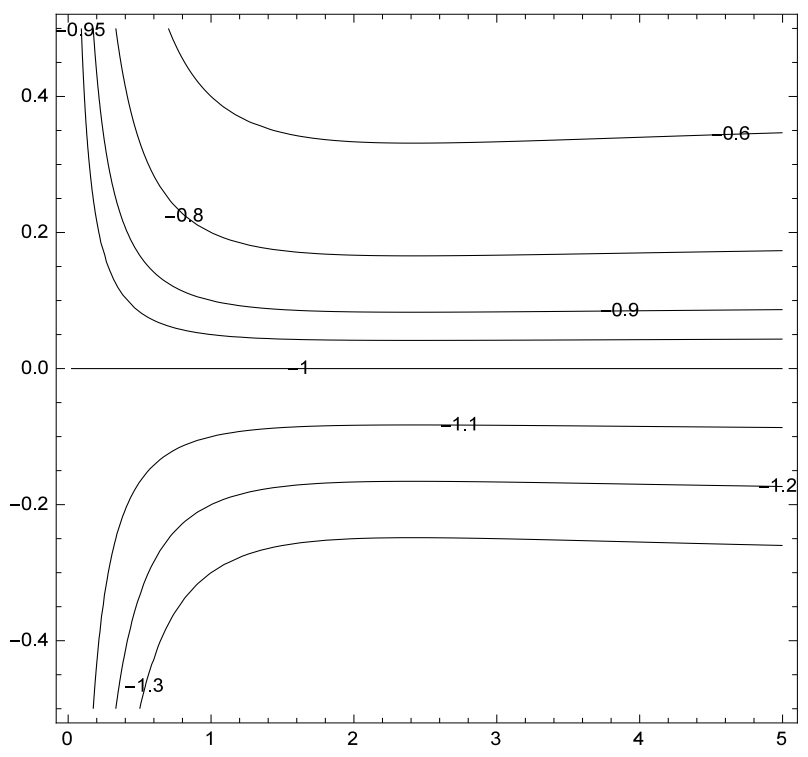

FIG. 1: Contour plots for the parametric model $A$ (BarbozaAlcaniz parametrization) of (3) is presented for $w_{0}=-1$. Horizontal axis presents the redshift history while the vertical axis takes the values of the constant $w_{a}$.

$\left.w_{x}=w_{x}(a(t))\right)$ while in particular we consider four nonlinear functions $w_{x}(a)$, where three of which have been proposed before in the litterature and we propose a new parameterization in this work.

The scope of our analysis is to study the viability of those models with the use of the recent cosmological data, and also to determine the best parameters of the models. Let us remark that throughout the work we set $a_{0}=1$.

\section{1. $\quad$ Model A}

As the first model we consider the Barboza-Alcaniz equation of state parameter 33] which has two free parameters

$$
w_{x}(z)=w_{0}+w_{a} \frac{z(1+z)}{1+z^{2}} .
$$

Parameter $w_{0}$ gives the value of $w_{x}$ at present, that is $w_{0}=w_{x}(z \rightarrow 0)$, while for large values of $z, w_{x}(z)$ becomes constant. Moreover, the parameter $w_{a}$ denotes the rate of change of the equation of state parameter today, that is, $w_{a}=\left.\frac{d w_{x}}{d z}\right|_{z \rightarrow 0}$. One can easily realize that this parametrization is divergence free.

In Fig. 1 the evolution of the the parametric model is presented in terms of the redshift and the second parameter $w_{a}$. 


\subsection{Model B}

A nonlinear model for the equation of state parmater of dark energy was proposed in [35. The functional form of the model as presented in [35] is

$$
w_{x}(z)=-1+\frac{1}{3}\left(\frac{\beta(1+z)}{\alpha+\beta(1+z)}\right)
$$

where parameters $\alpha$ and $\beta$ are positive real numbers. However, although the model contains two free parameters but it is easy to see that there is only one real free parameter.

In particular, we consider a natural generalization of the model and we consider the following functional form

$$
w_{x}(z)=w_{1}+\frac{1}{3}\left(\frac{(1+z)}{w_{2}+(1+z)}\right)
$$

where $w_{1}, w_{2}(\neq 0,-1)$, are the free parameters ${ }^{1}$. Now considering the present value of the dark energy equation of state, $w_{0}$, for the parametrization (4) one has $w_{0}=$ $w_{1}+\frac{1}{3\left(w_{2}+1\right)}$. However, we observe that for large redshifts the equation of state parameter for the model becomes constant in a similar way with that of the first model (3).

It is easy to see that using the current value of the dark energy equation of state $w_{0}$, the parametrization (4) can be recast as

$$
w_{x}(z)=\left(w_{0}-\frac{1}{3\left(w_{2}+1\right)}\right)+\frac{1}{3}\left(\frac{(1+z)}{w_{2}+(1+z)}\right),
$$

We note that the model (5) may diverge at finite time given by $z=-1-w_{2}$. However, we make it clear that for $w_{2}>0$ the model does not show any diverging nature in past or future. The model sustains its diverging character only for the negative values of $w_{2}$. Precisely, one can find that (5) could diverge at past or future, respectively for $w_{2}<-1$ or $-1<w_{2}<0$. Moreover, in Fig. 2 we present the evolution of the parametric equation of state parmeter in terms of the cosmic history and the free parameter $w_{2}$.

\subsection{Model C}

We introduce another model that was originally introduced in 36. We note that except the nonlinear character, the equation of state parameter depends on the energy density of the matter sector (cold dark matter plus baryons), that is,

\footnotetext{
${ }^{1}$ It must be mentioned that $w_{2}$ cannot take $0,-1$ because, for $w_{2}=0, w_{x}(z)$ becomes constant while for $w_{2}=-1$, the model diverges at $z=0$, which is unphysical.
}

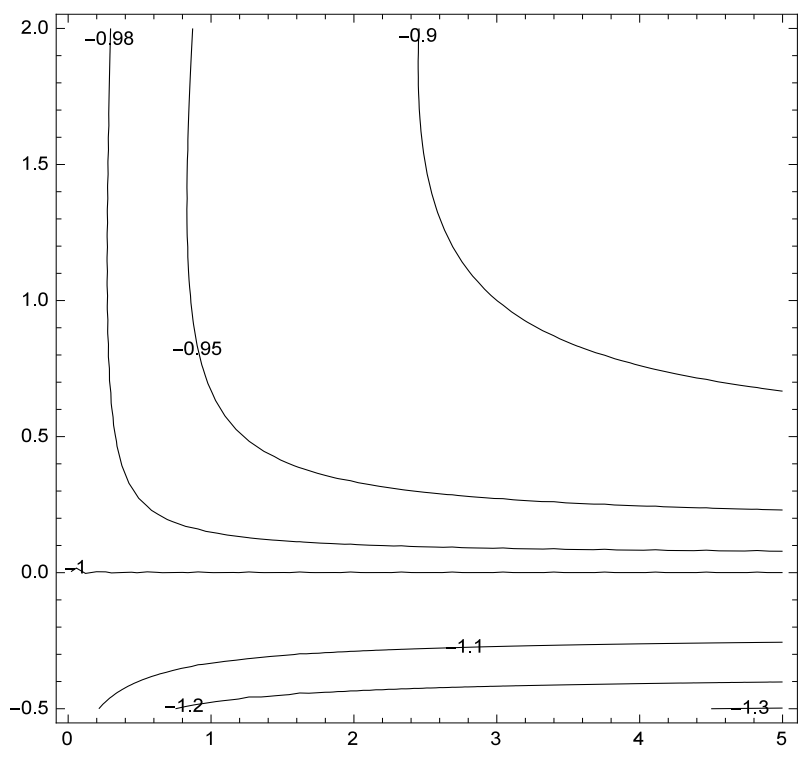

FIG. 2: Contour plots for the parametric model $B$ (Zhang et al. parametrization) of (5) is presented for the free parameter $w_{0}=-1$. Horizontal axis presents the redshift history while the vertical axis provides the second parameter $w_{2}$.

$$
w_{x}(z)=\frac{a_{1}+3\left(\Omega_{m 0}-1\right)-2 a_{1} z-a_{2}\left(z^{2}+2 z-2\right)}{3\left(1-\Omega_{m 0}+a_{1} z+2 a_{2} z+a_{2} z^{2}\right)},
$$

where $a_{1}, a_{2}$ are free constants and $\Omega_{m 0}$, is the current density parameter for matter sector.

One can observe that both for $a_{1}=a_{2}=0, w_{x}(z)=$ -1 . Thus, in order for $(6)$ to represent a dynamical equation of state for the dark energy model, one of the free parameters, namely $a_{1}, a_{2}$ must be nonzero. However, since $(6)$ is a rational function, so one may realize that the model could exhibit past or future singularities depending on the free parameters. Let us first consider the simplest cases when only one of $a_{1}$ and $a_{2}$ is zero. For instance, if we consider that $a_{2}=0$, but $a_{1} \neq 0$, we see that the model 60 gives a singularity at $z=-\left(\frac{1-\Omega_{0}}{a_{1}}\right)$. Now, this sigularity could be at past or present depending on the nature of $a_{1}$. For $a_{1}<0$, the singularity appears at $z>0$, that means a past singularity while for $a_{1}>0$, the singularity arises at $z<0$ menaing a future singularity. We consider the second possibility when $a_{1}=0$, but $a_{2} \neq 0$. Here two distinct possibilities arise - when $a_{2}^{2}-a_{2}\left(1-\Omega_{m 0}\right)<0$, we do not have any singularity but for $a_{2}^{2}-a_{2}\left(1-\Omega_{m 0}\right)>0$ we have singularities. Now for this second case, if $a_{2}<0$, then we always have a singularity given by $z=-1-\frac{1}{a_{2}} \sqrt{a_{2}^{2}-a_{2}\left(1-\Omega_{m 0}\right)}$. This singularity can be classified as past or future singularity whether $z>0$, or $-1<z<0$, respectively. On the other hand, for $a_{2}>0$, singularity exists for $a_{2}>\left(1-\Omega_{m 0}\right)$ and the singularity occurs at $z=-1+\frac{1}{a_{2}} \sqrt{a_{2}^{2}-a_{2}\left(1-\Omega_{m 0}\right)}$. One can similarly classify this singularity as described just above. Now we 


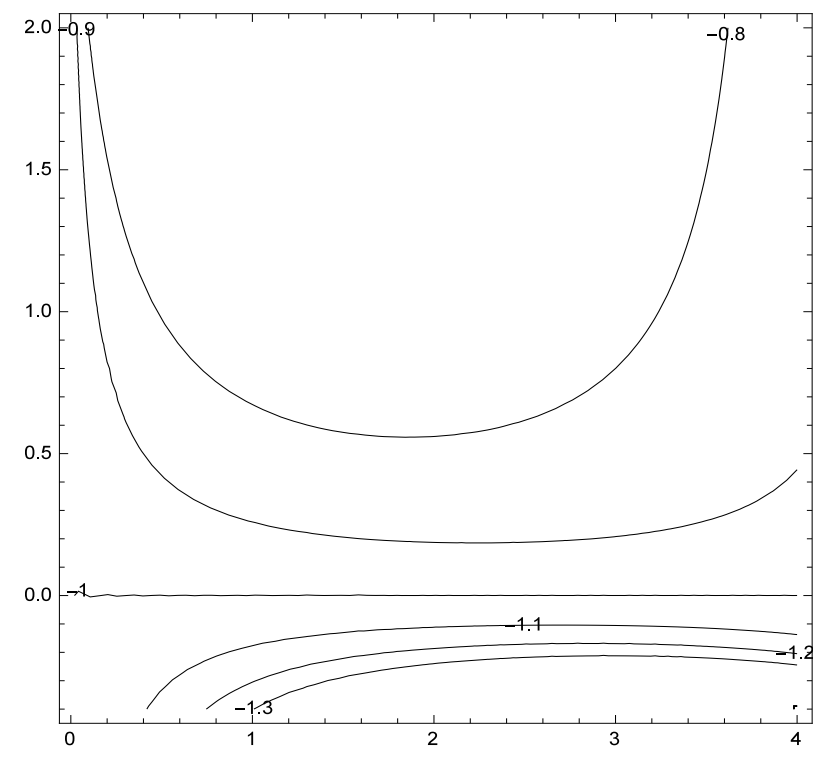

FIG. 3: Contour plots for the parametric model $C$ (Nesseris and Perivolaropoulos parametrization) of (6) is presented for the free parameter $\Omega_{m 0}=0.30$ and $w_{x}(z \rightarrow 0)=-1$. Horizontal axis presents the redshift history while the vertical axis provides the free parameter $a_{1}$.

consider the general case when $a_{1} \neq 0$ and $a_{2} \neq 0$. We notice that the parametrization could have real singularities if $\left(2 a_{2}+a_{1}\right)^{2}-4 a_{2}\left(1-\Omega_{m 0}\right)>0$. However, for $\left(2 a_{2}+a_{1}\right)^{2}-4 a_{2}\left(1-\Omega_{m 0}\right)<0$, one does not encouter with any real singularities in the above parametrization.

The evolution of this equation of state parameter is displayed in Fig. 3 for $\Omega_{m 0}=0.30$ and the constraint $w_{x}(a \rightarrow 1)=-1$.

\subsection{Model D}

We introduce the final model in this series having the following form

$$
w_{x}(z)=w_{0}+\frac{w_{b}}{1+z} \ln (1+z)
$$

where $w_{0}$ is the current value of $w_{x}(z)$ and $w_{b}$ is any free parameter. We note that for $z \rightarrow \infty, w_{x}(z) \rightarrow w_{0}$. One can see that at low redshifts, the model (7) recovers the CPL parametrization $w_{x}(z)=w_{0}-w_{b}\left(\frac{z}{1+z}\right)$. That means at very low redshifts, the evolution of (7) and the evolution of the CPL parametrization are equivalent.

In Fig. 4 we present the evolution of the parametric equation of state parmeter in terms of the cosmic history and the free parameter $w_{b}$.

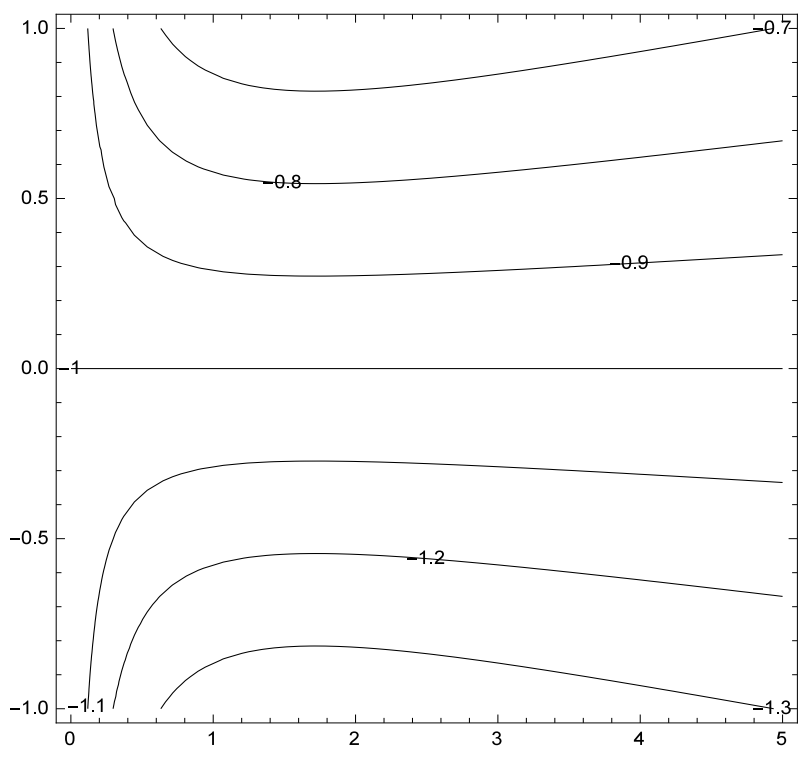

FIG. 4: Contour plots for the parametric model D (The new parametrization) of (7) is presented for $w_{0}=-1$. Horizontal axis presents the redshift history while the vertical axis takes the values of the constant $w_{b}$.

\section{DYNAMICAL DARK-ENERGY AT PERTURBATIVE LEVEL}

The most general scalar mode perturbation is characterized by the following metric [53, 55$]$

$$
\begin{gathered}
d s^{2}=-(1+2 \phi) d t^{2}+2 a \partial_{i} B d t d x+ \\
a^{2}\left[(1-2 \psi) \delta_{i j}+2 \partial_{i} \partial_{j} E\right] d x^{i} d x^{j}
\end{gathered}
$$

In this work we follow some standard formalism as described in Ref. 54, and adopt the synchronous gauge, that means we consider $\phi=B=0, \psi=\eta$, and $k^{2} E=-h / 2-3 \eta$. Using the above metric in consideration of the synchronous gauge, the conservation equations for the $i$-th component of the fluid are found to be

$$
\begin{aligned}
& \delta_{i}^{\prime}=-\left(1+w_{i}\right)\left(\theta_{i}+\frac{h^{\prime}}{2}\right)-3 \mathcal{H}\left(\frac{\delta P_{i}}{\delta \rho_{i}}-w_{i}\right) \delta_{i} \\
& \theta_{i}^{\prime}=-\mathcal{H}\left(1-3 w_{i}\right) \theta_{i}-\frac{w_{i}^{\prime}}{1+w_{i}} \theta_{i}+\frac{\delta P_{i} / \delta \rho_{i}}{1+w_{i}} k^{2} \delta_{i}-k^{2} \sigma .
\end{aligned}
$$

Here the prime is the derivative with respect to the conformal time; $\mathcal{H}=a^{\prime} / a$, is the conformal Hubble parameter; and the remaining quantities $\sigma, \delta_{i}, \theta_{i}$, are respectively denote the shear, density perturbation and the velocity pertubation. The DE perturbation equations fol- 


\begin{tabular}{|c|c|c|c|}
\hline Parameters & Priors & Mean with errors & Best fit \\
\hline$\Omega_{c} h^{2}$ & {$[0.01,0.99]$} & $0.1186_{-0.0013-0.0025}^{+0.0013+0.0024}$ & 0.1195 \\
\hline$\Omega_{b} h^{2}$ & {$[0.005,0.1]$} & $0.0223_{-0.0002-0.0003}^{+0.0001+0.0003}$ & 0.0223 \\
\hline $100 \theta_{M C}$ & {$[0.5,10]$} & $1.0406_{-0.0003-0.0006}^{+0.0003+0.0006}$ & 1.0406 \\
\hline$\tau$ & {$[0.01,0.8]$} & $0.0636_{-0.0173-0.0338}^{+0.0170+0.0341}$ & 0.0502 \\
\hline$n_{s}$ & {$[0.5,1.5]$} & $0.9753_{-0.0044-0.0085}^{+0.0044+0.0090}$ & 0.9718 \\
\hline $\ln \left(10^{10} A_{s}\right)$ & {$[2.4,4]$} & $3.0676_{-0.0335-0.0640}^{+0.0326+0.0661}$ & 3.0468 \\
\hline$w_{0}$ & {$[-2,0]$} & $-0.9589_{-0.0910-0.1796}^{+0.0871+0.1797}$ & -0.9112 \\
\hline$w_{a}$ & {$[-1,1]$} & $-0.1536_{-0.1623-0.3740}^{+0.2109+0.3705}$ & -0.2828 \\
\hline$\Omega_{m 0}$ & - & $0.3031_{-0.0091-0.0158}^{+0.0079+0.0168}$ & 0.3051 \\
\hline$\sigma_{8}$ & - & $0.8197_{-0.0132-0.0271}^{+0.0144+0.0268}$ & 0.8177 \\
\hline$H_{0}$ & - & $68.3496_{-0.8664-1.6475}^{+0.8667+1.651}$ & 68.3179 \\
\hline$\chi_{\min }^{2}$ & - & - & 13720.556 \\
\hline
\end{tabular}

TABLE I: The table summarizes the observational constraints of the free and derived parameters of the Model A (BarbozaAlcaniz model) for the combined observational data Planck $T T, T E, E E+l o w T E B+J L A+B A O+R S D+W L+C C$ $+H_{0}$. Here, $\Omega_{m 0}=\Omega_{c 0}+\Omega_{b 0}$.

low

$$
\begin{aligned}
\delta_{x}^{\prime} & =-\left(1+w_{x}\right)\left(\theta_{x}+\frac{h^{\prime}}{2}\right)-3 \mathcal{H} w_{x}^{\prime} \frac{\theta_{x}}{k^{2}} \\
& -3 \mathcal{H}\left(c_{s}^{2}-w_{x}\right)\left[\delta_{x}+3 \mathcal{H}\left(1+w_{x}\right) \frac{\theta_{x}}{k^{2}}\right], \\
\theta_{x}^{\prime} & =-\mathcal{H}\left(1-3 c_{s}^{2}\right) \theta_{x}+\frac{c_{s}^{2}}{1+w_{x}} k^{2} \delta_{x}
\end{aligned}
$$

where without any loss of generality and simplicity we assume $\sigma=0 ; c_{s}^{2}$, is the physical sound speed in the rest frame which we set to be unity to avoid any kind of unphysical behaviour. The perturbation equations for the other components, namely, the baryons, radiation, and pressureless dark matter follow the standard equations as described in [53 55].

\section{OBSERVATIONAL DATA SETS}

To constrain the four parametric dark energy models we have used the latest astronomical data from several sources. In the following we briefly describe the employed data sets and their sources.

- The Cosmic Microwave Background data (CMB) given by the Planck 2015 collaboration [40, 41 have been used here. Here we combine the likelihoods $C_{l}^{T T}, C_{l}^{E E}, C_{l}^{T E}$ in addition to the low-l polarization data. We identify this data with the notation $C_{l}^{T E}+C_{l}^{E E}+C_{l}^{B B}$.

- The joint light curve (JLA) sample [42] provides with 740 Supernovae Type Ia (SNIa) data in the redshift range $z \in[0.01,1.30]$.

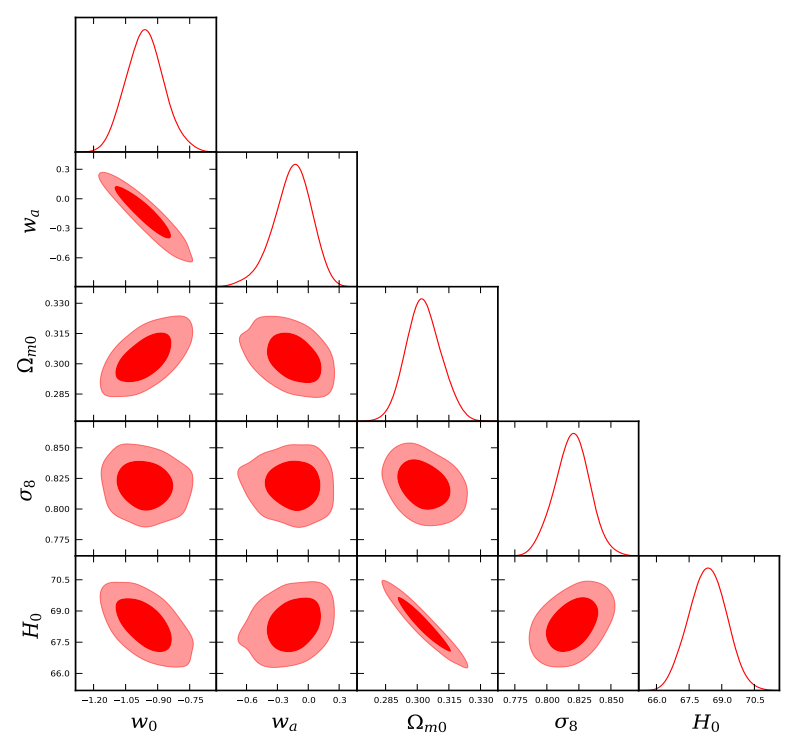

FIG. 5: The figure displays the $68.3 \%$ and $95.4 \%$ confidencelevel contour plots for different combinations of the model parameters of the Barboza-Alcaniz parametrization (3) using the

\begin{tabular}{|c|c|c|c|}
\hline Parameters & Priors & Mean with errors & Best fit \\
\hline$\Omega_{c} h^{2}$ & {$[0.01,0.99]$} & $0.1182_{-0.0013-0.0023}^{+0.0012+0.0023}$ & 0.1183 \\
\hline$\Omega_{b} h^{2}$ & {$[0.005,0.1]$} & $0.0223_{-0.0002-0.0003}^{+0.0001+0.0003}$ & 0.0223 \\
\hline $100 \theta_{M C}$ & {$[0.5,10]$} & $1.0406_{-0.0003-0.0006}^{+0.0003+0.0006}$ & 1.0408 \\
\hline$\tau$ & {$[0.01,0.8]$} & $0.0662_{-0.0182-0.0324}^{+0.0168+0.0344}$ & 0.0688 \\
\hline$n_{s}$ & {$[0.5,1.5]$} & $0.9762_{-0.0043-0.0075}^{+0.0039+0.0081}$ & 0.9787 \\
\hline $\ln \left(10^{10} A_{s}\right)$ & {$[2.4,4]$} & $3.0721_{-0.0349-0.0636}^{+0.0328+0.0668}$ & 3.0799 \\
\hline$w_{0}$ & {$[0,2]$} & $-1.0449_{-0.0322-0.0717}^{+0.0361+0.0677}$ & -1.0416 \\
\hline$w_{2}$ & {$[-3,3]$} & $0.2254_{-0.2254-0.2254}^{+0.0509+0.3364}$ & 0.0220 \\
\hline$\Omega_{m 0}$ & - & $0.2991_{-0.0079-0.0142}^{+0.0072+0.0147}$ & 0.2971 \\
\hline$\sigma_{8}$ & - & $0.8198_{-0.0139-0.0276}^{+0.0147+0.0266}$ & 0.8269 \\
\hline$H_{0}$ & - & $68.7052_{-0.8120-1.6566}^{+0.8192+1.6440}$ & 68.9673 \\
\hline$\chi_{\min }^{2}$ & - & - & 13722.266 \\
\hline
\end{tabular}
combined analysis Planck TT, TE, EE + lowTE $\bar{B}+J L A+$ $B A O+R S D+W L+C C+H_{0}$.

TABLE II: The table summarizes the observational constraints of the free and derived parameters of Model B (5) (Zhang et al. parametrization) for the combined observational data Planck TT, TE, EE + lowTEB + JLA + BAO+RSD $+W L+C C+H_{0}$. We note that, $\Omega_{m 0}=\Omega_{c 0}+\Omega_{b 0}$.

- The Baryon acoustic oscillations (BAO) distance measurements from various collaborations are used. In particular, for the $\mathrm{BAO}$ data we consider the estimated ratio of $r_{s} / D_{V}$ as a 'standard ruler' in which $r_{s}$ is the comoving sound horizon at the baryon drag epoch and $D_{V}$ is the effective distance determined by the angular diameter distance $D_{A}$ and Hubble parameter $H$ taking the form $D_{V}(z)=$ $\left[(1+z)^{2} D_{A}(a)^{2} \frac{z}{H(z)}\right]^{1 / 3}$. For this data set, we use four BAO points, namely the 6dF Galaxy Survey 


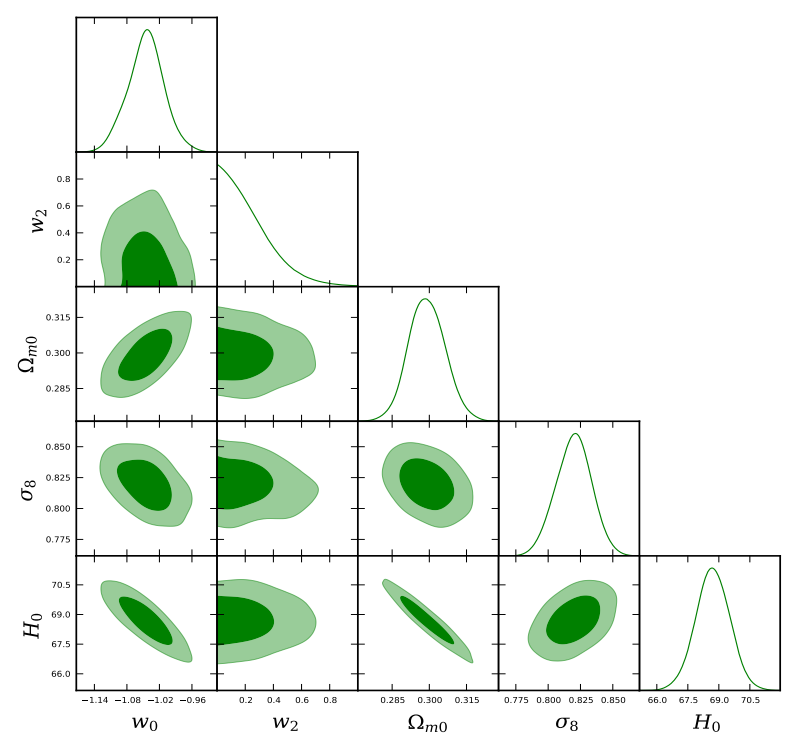

FIG. 6: The figure displays the $68.3 \%$ and $95.4 \%$ confidencelevel contour plots for different combinations of the model parameters of the Zhang et al. parametrization (5) using the combined analysis Planck TT, TE, EE + lowTEB + JLA + $B A O+R S D+W L+C C+H_{0}$.

\begin{tabular}{cccc}
\hline \hline Parameters & Priors & Mean with errors & Best fit \\
\hline$\Omega_{c} h^{2}$ & {$[0.01,0.99]$} & $0.1182_{-0.0013-0.0024}^{+0.0012+0.0025}$ & 0.1186 \\
$\Omega_{b} h^{2}$ & {$[0.005,0.1]$} & $0.0223_{-0.0002+-00003}^{+0.0002-0.0003}$ & 0.0222 \\
$100 \theta_{M C}$ & {$[0.5,10]$} & $1.0406_{-0.0003-0.0006}^{+0.0003+0.0006}$ & 1.0405 \\
$\tau$ & {$[0.01,0.8]$} & $0.0687_{-0.0165-0.0344}^{+0.0166+0.0328}$ & 0.0604 \\
$n_{s}$ & {$[0.5,1.5]$} & $0.9761_{-0.0042-0.0084}^{+0.0045+0.0083}$ & 0.9768 \\
$\ln \left(10^{10} A_{s}\right)$ & {$[2.4,4]$} & $3.0759_{-0.0325-0.0627}^{+0.0324}$ & 3.0658 \\
\hline$a_{1}$ & {$[-1,0]$} & $-0.1379_{-0.0548-0.1613}^{+0.1107+0.1379}$ & -0.1113 \\
$a_{2}$ & {$[0,1]$} & $0.0244_{-0.0244-0.0244}^{+0.0058+0.0366}$ & 0.0138 \\
\hline$\Omega_{m 0}$ & - & $0.2984_{-0.0073-0.0144}^{+0.0074+0.0137}$ & 0.2990 \\
$\sigma_{8}$ & - & $0.8195_{-0.0135+0.0252}^{+0.0130-0.0268}$ & 0.8222 \\
$H_{0}$ & - & $68.7759_{-0.8318-1.5894}^{+0.7928+1.5598}$ & 68.7964 \\
\hline$\chi_{\text {min }}^{2}$ & - & \multicolumn{2}{c}{13723.394} \\
\hline \hline
\end{tabular}

TABLE III: The table summarizes the observational constraints of the free and derived parameters of the Model $C$ (Nesseris-Perivolaropoulos parametrization) for the combined observational data Planck TT, TE, EE + lowTEB + JLA + $B A O+R S D+W L+C C+H_{0}$. We note that, $\Omega_{m 0}=$ $\Omega_{c 0}+\Omega_{b 0}$.

(6dFGS) measurement at $z_{\text {eff }}=0.106$ [3], the Main Galaxy Sample of Data Release 7 of Sloan Digital Sky Survey (SDSS-MGS) at $z_{\text {eff }}=0.15$ 44, and the CMASS and LOWZ samples from the latest Data Release 12 (DR12) of the Baryon Oscillation Spectroscopic Survey (BOSS) at $z_{\text {eff }}=0.57$ 45] and $z_{\text {eff }}=0.32$ [45.

- We use the Redshift space distortion (RSD) data from two observational surveys which include the

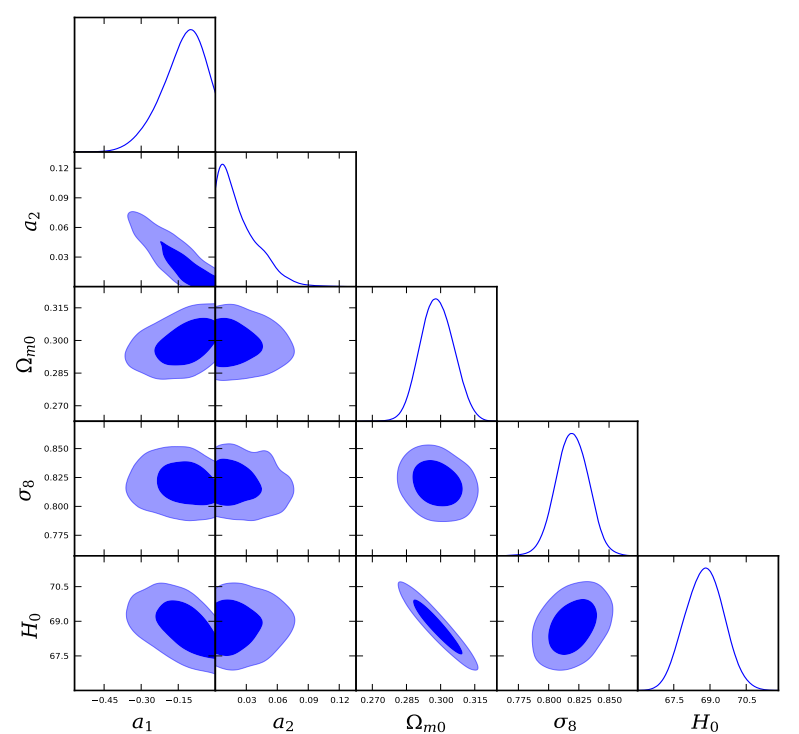

FIG. 7: The figure displays the $68.3 \%$ and $95.4 \%$ confidencelevel contour plots for different combinations of the model parameters of the Nesseris-Perivolaropoulos parametrization (6) using the combined analysis Planck TT, TE, EE + lowTEB $+J L A+B A O+R S D+W L+C C+H_{0}$.

\begin{tabular}{cccc}
\hline \hline Parameters & Priors & Mean with errors & Best fit \\
\hline$\Omega_{c} h^{2}$ & {$[0.01,0.99]$} & $0.1182_{-0.0012-0.0023}^{+0.0011+0.0023}$ & 0.1186 \\
$\Omega_{b} h^{2}$ & {$[0.005,0.1]$} & $0.0223_{-0.0001+0.0003}^{+0.0001+0003}$ & 0.0223 \\
$100 \theta_{M C}$ & {$[0.5,10]$} & $1.0406_{-0.0003-0.0006}^{+0.0003+0.0006}$ & 1.0405 \\
$\tau$ & {$[0.01,0.8]$} & $0.0677_{-0.0179-0.0317}^{+0.0161+0.0339}$ & 0.0616 \\
$n_{s}$ & {$[0.5,1.5]$} & $0.9762_{-0.0043-0.0077}^{+0.0039+0.0080}$ & 0.9732 \\
$\ln \left(10^{10} A_{s}\right)$ & {$[2.4,4]$} & $3.0741_{-0.0344-0.0677}^{+0.0309+0.0624}$ & 3.0669 \\
\hline$w_{0}$ & {$[-2,0]$} & $-1.0533_{-0.0321-0.06398}^{+0.0362+0.0639}$ & -1.0376 \\
$w_{b}$ & {$[0,2]$} & $0.0971_{-0.0971-0.0971}^{+0.0160+0.1694}$ & 0.0201 \\
\hline$\Omega_{m 0}$ & - & $0.2987_{-0.0070-0.0143}^{+0.0071+0.0145}$ & 0.3011 \\
$\sigma_{8}$ & - & $0.8212_{-0.0147-0.0278}^{+0.0125+0.0243}$ & 0.8189 \\
$H_{0}$ & - & $68.7539_{-0.8010-1.5505}^{+0.7703+1.6054}$ & 68.5523 \\
\hline$\chi_{\min }^{2}$ & - & & - \\
\hline \hline
\end{tabular}

TABLE IV: The table summarizes the observational constraints of the free and derived parameters of the Model D (The new parametrization) for the combined observational data Planck TT, TE, EE + lowTEB + JLA + BAO + RSD $+\mathrm{WL}+\mathrm{CC}+H_{0}$. We note that, $\Omega_{m 0}=\Omega_{c 0}+\Omega_{b 0}$.

CMASS sample with an effective redshift of $z_{\text {eff }}=$ 0.57 [45] and the LOWZ sample with an effective redshift of $z_{\text {eff }}=0.32$ [45].

- Moreover, we consider the weak gravitational lensing (WL) data from blue galaxy sample compliled from Canada-France-Hawaii Telescope Lensing Survey (CFHTLenS) 46, 47.

- Finally, cosmic chronometers (CC) plus the local value of the Hubble parameter $\left(C C+H_{0}\right)$ are 


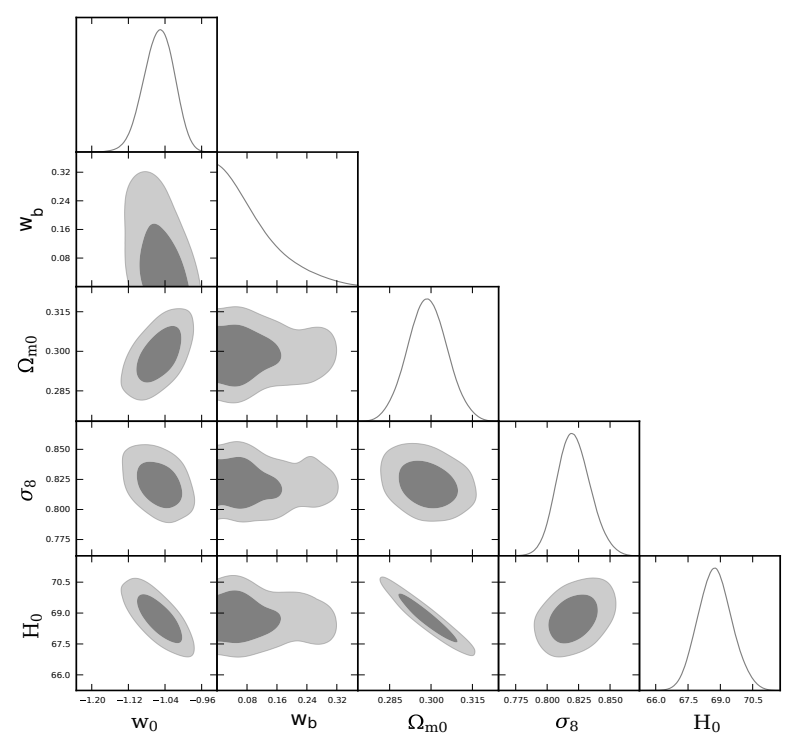

FIG. 8: The figure displays the $68.3 \%$ and $95.4 \%$ confidencelevel contour plots for different combinations of the model parameters of the new parametrization (7) using the combined analysis Planck TT, TE, EE + lowTEB + JLA+BAO+ $R S D+W L+C C+H_{0}$.

added with the previous data sets. The cosmic chronometers data compile 30 measurements of the Hubble parameter values in the redshift interval $0<z<2$ [8] and they are determined through a model independent manner, hence, they are believed to provide with crucial information from the cosmological model. The local value of the Hubble parameter from the Hubble Space Telescope yields $H_{0}=73.02 \pm 1.79 \mathrm{~km} / \mathrm{s} / \mathrm{Mpc}$ with $2.4 \%$ precision as determined recently in [49].

\section{RESULTS OF THE OBSERVATIONAL CONSTRAINTS}

Let us summarize the main observational constraints extracted from the parametric dark energy models using the combined astronomical data Planck TT, TE, EE + lowTEB + JLA + BAO+RSD + WL +CC+ $H_{0}$. The analysis is based on the total likelihood $\mathcal{L} \propto e^{-\chi_{\text {tot }}^{2} / 2}$, where $\chi_{\text {tot }}^{2}=\sum_{j} \chi_{j}^{2}$, and $j$ runs over all the data sets such as CMB (Planck TT, TE, EE + lowTEB), JLA, BAO, $R S D, W L, C C$ and $H_{0}$. For the statistical analysis, we use the Markov Chain Monte Carlo package cosmomc, a Metropolis-Hastings algorithm [50, 51] which extracts the cosmological constraints in an efficient way following a convergence criteria of the model parameters based on the Gelman-Rubin statistics [52]. Precisely, for each dark energy model, we modify a fast Boltzmann code known as CAMB (Cosmic Anisotropy Microwave Background) within the cosmomc pacakge to solve the evolution equations and consequently, the $\chi_{\text {tot }}^{2}$ function for the com-

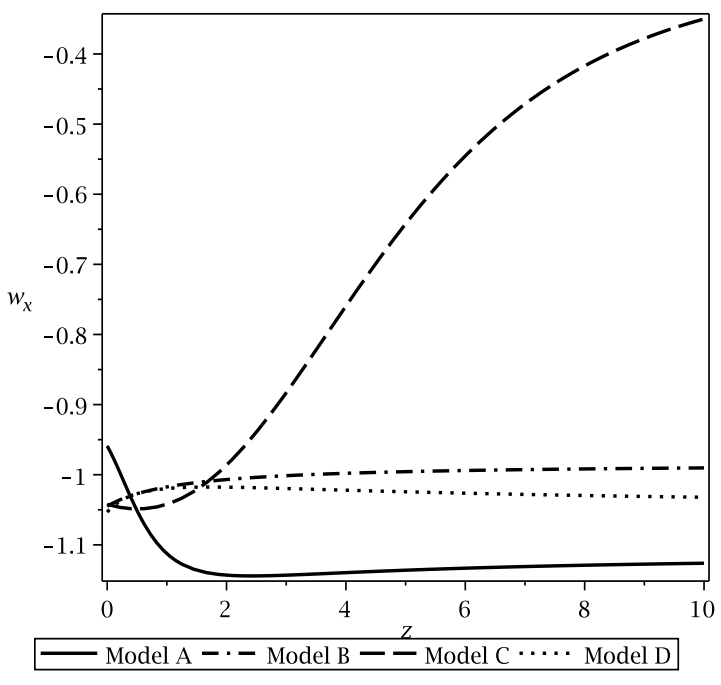

FIG. 9: The evolution of the dark energy equation of state for models $A-D$ using the mean values from Planck TT, TE, EE + lowTEB $+J L A+B A O+R S D+W L+C C+H_{0}$. Solid line is for model A, dash-dot line for model $\mathrm{B}$, dash-dash line for model $\mathrm{C}$ and dot-dot line is for model D. From the plot it can be seen that all the models cross the phantom divine line in the past while only model A provides an equation of state parameter with value bigger than minus one at the present time.

bined data set. Finally, calling cosmomc we explore the parameters space for the models. In all parametric models, we have the following eight-dimensional parameters space

$$
\mathcal{P} \equiv\left\{\Omega_{b} h^{2}, \Omega_{c} h^{2}, 100 \theta_{M C}, \tau, P_{1}, P_{2}, n_{s}, \log \left[10^{10} A_{s}\right]\right\}
$$

where $P_{1}, P_{2}$ are the model parameters and the remaining parameters $\left(\Omega_{b} h^{2}, \Omega_{c} h^{2}, 100 \theta_{M C}, \tau, n_{s}, A_{s}\right)$ are respectively the baryons density, cold dark matter density, ratio of sound horizon to the angular diameter distance, optical depth, scalar spectral index, and the amplitude of the initial power spectrum. In the following we describe the results for each model.

- Model A: We constrain this parametrization using the combined observational data mentioned above. The Table I summarizes the observational constraints of the free parameters $\left(w_{0}, w_{a}\right)$ of this model as well as the derived parameters $\left(\sigma_{8}, \Omega_{m 0}\right.$, $\left.H_{0}\right)$ and the Figure 5 displays the $68.3 \%(1 \sigma)$ and 95.4\% $(2 \sigma)$ confidence-level contour plots for several combinations of the free and derived parameters. Our analysis shows that the best fit and the mean value of the dark energy equation of state, $w_{x}$, for this parametrization show its quintessential nature, however, one cannot exclude the possibility of the dark energy equation of state to be phantom. In fact at $1 \sigma$ lower confidence-level, 
$w_{x}<-1$, but that is very close to the cosmological constant $w_{x}=-1$. However, we note that the observed tension between the different values of $H_{0}$ reported from the local $\left(H_{0}=67.27 \pm 0.66\right.$ $\left.\mathrm{Km} \mathrm{s}^{-1} \mathrm{Mpc}^{-1}\right)$ 49] and the global measurements $\left(H_{0}=73.24 \pm 1.74 \mathrm{Km} \mathrm{s}^{-1} \mathrm{Mpc}^{-1}\right)$ [6] may be reconciled for this model. This might be considered to be one of the interesting properties in the dynamical DE models. We finally note that the estimation of $\sigma_{8}\left(=0.8197_{-0.0132}^{+0.0144}\right.$ at $68.3 \%$ confidence-level) for this model is close to the Planck's $\Lambda$ CDM based estimation, in particular when lensing is added to either Planck TT+lowP or Planck TT, TE, EE+lowP. Precisely the estimated values of $\sigma_{8}$ are $\sigma_{8}=0.8149 \pm 0.0093$ at $68.3 \%$ confidence-level (Planck TT+lowP+lensing) [6] and $\sigma_{8}=0.8150 \pm 0.0087$ at $68.3 \%$ confidencelevel (Planck TT, TE, EE+lowP+lensing) 6]. The addition of external data $\left(\mathrm{BAO}+\mathrm{JLA}+H_{0}\right)$ to both Planck TT+lowP+lensing or Planck TT, TE, EE+lowP+lensing does not offer any significant changes to $\sigma_{8}$, see [6].

- Model B: We summarize the observational constraints for this model in Table II and in Figure 6 we show the corresponding contours at $68.3 \%$ and $95.4 \%$ confidence-levels. For the analysis we have used the same observational data as mentioned. Here we see that both best fit and the mean values of the dark energy equation of state $w_{x}$, are phantom! In fact, within $1 \sigma$ confidence-level, the dark energy equation of state remains phantom, i.e. $w_{x}<-1$, however, within $2 \sigma$ confidence-level it is quintessential. One can further notice that the model can be considered to reconcile the current tension on $H_{0}$ within a confidence level slightly greater than $1 \sigma$. Further, we also see that the value of $\sigma_{8}$ for this model is very similar to the estimated value of $\sigma_{8}$ for Model $A$ and also very close to the Planck's $\Lambda$ CDM based estimation in presence of lensing [6].

- Model C: As far as the authors are concerned with [36], this model has not been constrained yet due to its very complicated nature. Using the monte-carlo simulation and the same observational data, we found that the model can be constrained. Thus, we present the observational constraints of this model summarized in Table III while Figure 7 shows the $1 \sigma$ and $2 \sigma$ confidence-level contour plots for several combinations of the free and derived parameters. Using the constrained values of the free parameters $a_{1}$ and $a_{2}$ and the derived parameter $\Omega_{m 0}$, the current value of the dark energy equation of state, $w_{0}$, can be reproduced. The mean and the best fit values of $w_{0}$ up to 4 places of decimal can be found to be -1.0426 , and -1.0401 , respectively, which show that both mean and the best fit values of the dark energy equation of state for this model are very close to the cosmological constant boundary ' -1 '. The current tension on $H_{0}$ can also be relieved in this dynamical model in the confidence level greater than $1 \sigma$. Concerning the estimated value of $\sigma_{8}$, this model also follows similar trend to that of Model $A$ and $B$ and hence Planck's estimation in presence of lensing [6].

- Model D: This is a new model proposed in this work. Using the same combined analysis applied to the previous models we constrain this model with results summarized in Table IV and the corresponding contour plots at $1 \sigma$ and $2 \sigma$ confidence-levels displayed in Figure 8. We see that the current value of the dark energy equation of state, $w_{0}$, for this model crosses the phantom divide line, moreover, within $1 \sigma$ confidence-level, $w_{0}<-1$. However, the results on $w_{0}$ show that within $2 \sigma$ confidence-level, the behaviour of $w_{0}$ becomes quintessential. Concerning the current tension on $H_{0}$, this model follows a similar trend to Models $B$ and $C$. However, one can see that the model allows a slight greater value of $\sigma_{8}$ in compared to its value obtained for the Models $A-C$ as well as from the $\Lambda$-cosmology [6]. One can see that Model $D$ is slightly different from the other three models, but however, the difference is not much significant.

We close this section with Figure 9 that shows the behaviour of $w_{x}(z)$ for models $A-D$ using the mean values of the free and derived parameters from the said analysis. The figure shows that at late time, the models $B-D$ cross the cosmological constant boundary leading to a phantom dark energy dominated universe while for model $A$ the dark energy equation of state still lies just above the cosmological constant line, which hints for a quintessence dark energy dominated universe.

\section{SUMMARY AND CONCLUSIONS}

The theory of dark energy has been one of the central themes for describing the current accelerating phase of the universe. From the simplest cosmological constant to several complicated dark energy models are included in this category. Parametric dark energy models are one of them where the evolution of the dark energy models are described through their equations of state. In this work we consider some parametric dark energy models, namely, Model $A$, Model $B$, Model $C$ and Model $D$ in order to understand the late-evolution of the universe. Among these models although Model $A$ has been tested earlier with the observational data while the others are not, but still we tested Model $A$ again in order to update its observational constraints from the latest observational data that we employ in this work. To provide a better constarints on the models, we consider the large scale behaviour of the models and examine the models 


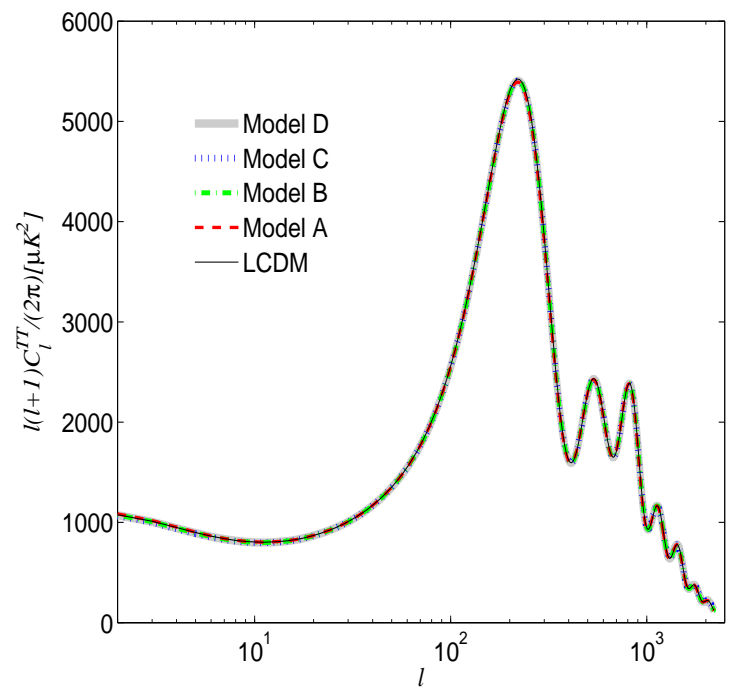

FIG. 10: Angular CMB temperature power spectra for the $\Lambda$ cosmology (LCDM) and the parametric dark energy models $A-D$ have been displayed using the mean values of the model parameters from Planck TT, TE, EE + lowTEB + JLA + $B A O+R S D+W L+C C+H_{0}$. We see that the curves in the plot are almost indistinguishable from each other meaning their closeness to each other.

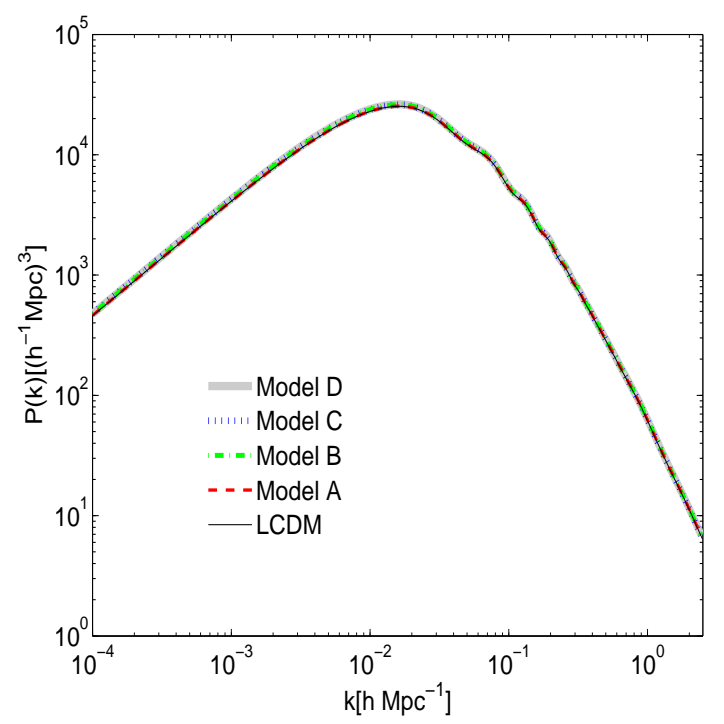

FIG. 11: Matter power spectra for the $\Lambda$-cosmology (LCDM) and the parametric dark energy models $A-D$ using the mean values of the model parameters from Planck TT, TE, EE + lowTEB $+J L A+B A O+R S D+W L+C C+H_{0}$. The curves in the plot cannot be distinguished from each other which means that the models at large scales are very close to each other.
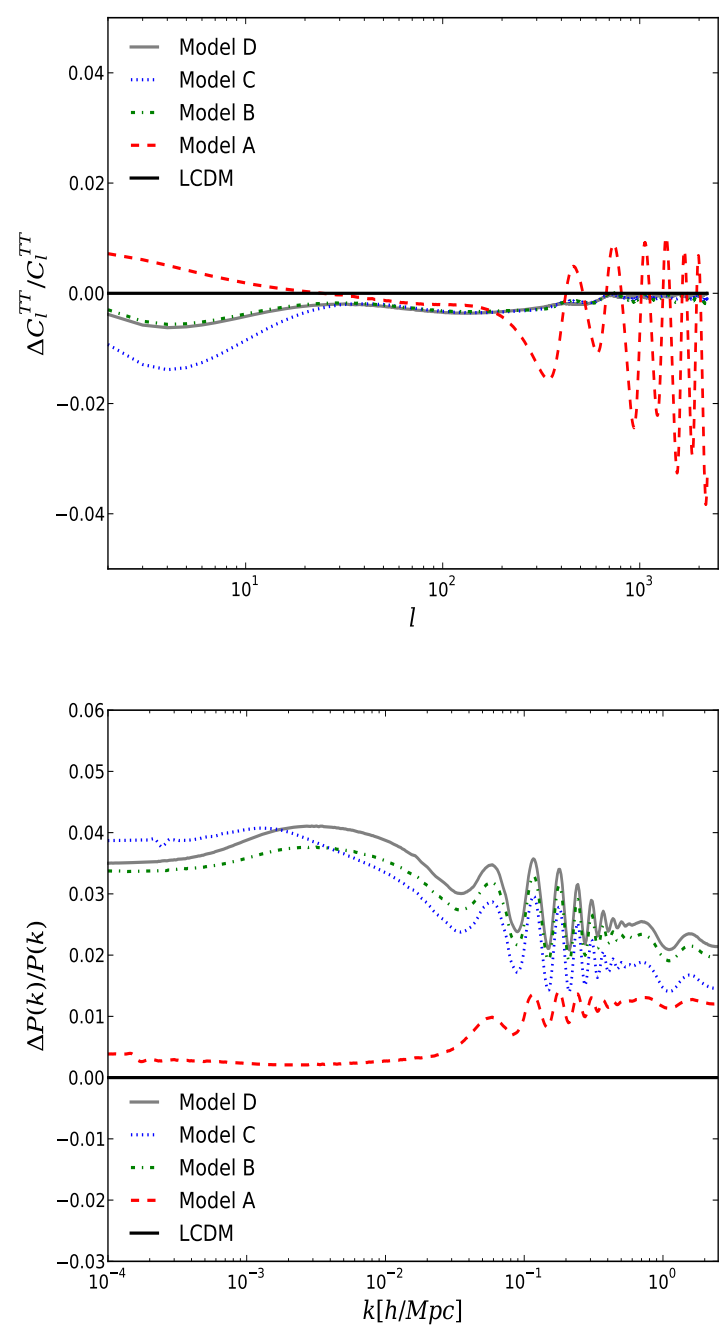

FIG. 12: The figure displays the relative deviations in the $C M B T T$ spectra $\left(\Delta C_{l}^{T T} / C_{l}^{T T}\right)$ and the matter power spectra $\left(\Delta P_{k}^{T T} / P_{k}^{T T}\right)$ from the flat $\Lambda$-cosmology (LCDM) using the combined analysis Planck TT, TE, EE + lowTEB + $J L A+B A O+R S D+W L+C C+H_{0}$. Here, $\Delta C_{l}^{T T}=$ $\left.C_{l}^{T T}\right|_{\text {model }}-\left.C_{l}^{T T}\right|_{L C D M}$ and $C_{l}^{T T}=\left.C_{l}^{T T}\right|_{L C D M}$. Similarly, $\Delta P(k)=\left.P(k)\right|_{\text {model }}-\left.P(k)\right|_{L C D M}$ and $P(k)=\left.P(k)\right|_{L C D M}$. One can notice that the parametric dark energy models in (3), (4), (6) and (7) have deviations from the flat $\Lambda$-cosmology, however, from the deviations as seen in both the plots one can conclude that the models are not far from the $\Lambda$-cosmology.

with the combined analysis of cosmic microwave background radiation, Supernove Type Ia, baryon acoustic oscillations, redshift space distortion data, weak gravitational lensing data, cosmic chronometers plus the local Hubble constant measured at $2.4 \%$ precision. Finally, we use the Markov Chain Monte Carlo package cosmomc, a Metropolis-Hastings algorithm [50, 51] to fit all the models.

Our analysis shows that at present epoch the Models $B, C$ and $D$ may allow the dark energy equation of state 
to cross the cosmological constant boundary ' $w_{x}=-1$ ', however, the equation of state for those models always stays very close to ' -1 '. For Model $A$ the dark energy equation of state at present is quintessential. Moreover, we find that within $68.3 \%$ confidence region, Model $A$ can alleviate the current tension on $H_{0}$ as suggested from the local [49] and global measurements [6] while the other models allow such property in a greater confidence level than $68.3 \%$. We have also found that the values of $\sigma_{8}$ for all models are almost similar and they are in agreement with the $\Lambda$ CDM based Planck's estimation [6], although for model $D$, a slightly higher value of $\sigma_{8}$ is preferred which however is not a serious issue to make a strong comment. In summary we find that the observational constraints on the models suggest that the current value of the dark energy equation of state is close to $w_{x}=-1$, but effectively the models differ from that of $\Lambda$-cosmology for non-null values of $w_{a}, w_{2}, w_{b}, a_{1}$ and $a_{2}$.

Furthermore, using the same observational data described above, we derive that $\chi_{\min }^{2}=13723.806$ $(\Lambda \mathrm{CDM}), \quad \chi_{\min }^{2}=13720.556($ Model $A), \chi_{\min }^{2}=$ 13722.266 (Model B), $\chi_{\min }^{2}=13723.394($ Model $C$ ) and $\chi_{\min }^{2}=13722.95($ Model $D)$. We observe that all the models fit the data in a similar way. It is important to mention that the parametric dark energy models have more degrees of freedom in compared to the $\Lambda$ cosmology. However, by using the Akaike information criterion [56, 57] to study the relation between the models with different degrees of freedom we can say that those models are statistically equivalent, at least with the current observational data.

Last but not least, in Figs. 10 and 11 we respectively show the angular CMB tempertaure power spectra and the matter power spectra for the parametric dark energy models in compared to the flat $\Lambda$-cosmology from which we observe that the parametric models are very close to each other as well as also with the $\Lambda$-cosmology. However, since the models are dynamical and different from one another, it is anticipated that they should exhibit deviation from each other (whatever small it could be) as well as from the $\Lambda$-cosmology. We note that the differences of the models are not detected from Fig. 10 and Fig. 11 while from the Fig. 12 measuring the relative deviations of the models to that of the $\Lambda$-cosmology in terms of the CMB TT spectra and matter power spectra, one can see that the models have deviations from each other as well as from $\Lambda$-cosmology. That is an expected result because in the past those parametric models should almost have a constant value for the equation of state parameter.

\section{Acknowledgements}

The authors thank the referee for some essential comments to improve the work. The work of WY has been supported by the National Natural Science Foundation of China under Grants No. 11705079 and No. 11647153. SP acknowledges the partial support from SERB-NPDF (File No. PDF/2015/000640). AP acknowledges the financial support of FONDECYT grant no. 3160121. The authors acknowledge the use of publicly available Monte Carlo Markov Chain package cosmomc.
[1] A. G. Riess et al. [Supernova Search Team], Astron. J. 116, 1009 (1998), astro-ph/9805201.

[2] S. Perlmutter et al. [Supernova Cosmology Project Collaboration], Astrophys. J. 517, 565 (1999), astro$\mathrm{ph} / 9812133$.

[3] M. Tegmark et al. [SDSS Collaboration], Astrophys. J. 606, 702 (2004), astro-ph/0310725.

[4] D. J. Eisenstein et al. [SDSS Collaboration], Astrophys. J. 633, 560 (2005), astro-ph/0501171.

[5] C. L. Bennett et al. [WMAP Collaboration], Astrophys. J. Suppl. 208, 20 (2013), arXiv:1212.5225 [astroph.CO]].

[6] P. A. R. Ade et al. [Planck Collaboration], Astron. Astrophys. 594, A13 (2016), arXiv:1502.01589 [astro-ph.CO]].

[7] E. J. Copeland, M. Sami and S. Tsujikawa, Int. J. Mod. Phys. D 15, 1753 (2006), hep-th/0603057.

[8] B. Ratra and P. J. E. Peebles, Phys. Rev. D 37, 3406 (1988).

[9] S. M. Carroll, Phys. Rev. Lett. 81, 3067 (1998), astro$\mathrm{ph} / 9806099$.

[10] L. A. Urena-Lopez and T. Matos, Phys. Rev. D 62, 081302 (2000), astro-ph/0003364.

[11] A. Y. Kamenshchik, U. Moschella and V. Pasquier, Phys. Lett. B 511, 265 (2001), gr-qc/0103004.
[12] J. de Haro, J. Amorós and S. Pan, Phys. Rev. D 93, 084018 (2016), arXiv:1601.08175 [gr-qc]].

[13] G. S. Sharov, JCAP 1606, 023 (2016), arXiv:1506.05246 [gr-qc]].

[14] W. Yang, R. C. Nunes, S. Pan and D. F. Mota, Phys. Rev. D 95, 103522 (2017), arXiv:1703.02556 [astroph.CO]].

[15] W. Yang, S. Pan and J. D. Barrow, accepted in Phys. Rev. D, arXiv:1706.04953 [astro-ph.CO].

[16] S. Nojiri, S. D. Odintsov and V. K. Oikonomou, Phys. Rept. 692, 1 (2017), arXiv:1705.11098 [gr-qc]].

[17] A. De Felice and S. Tsujikawa, Living Rev. Rel. 13, 3 (2010), arXiv:1002.4928 [gr-qc]].

[18] B. Li and J. D. Barrow, Phys. Rev. D 75, 084010 (2007), gr-qc/0701111.

[19] A. Paliathanasis, Class. Quant. Grav. 33, 075012 (2016), arXiv:1512.03239 [gr-qc]].

[20] T. P. Sotiriou and V. Faraoni, Rev. Mod. Phys. 82, 451 (2010) .

[21] A. Paliathanasis, J. D. Barrow and P. G. L. Leach, Phys. Rev. D 94, 023525 (2016), arXiv:1606.00659 [gr-qc]].

[22] Y. F. Cai, S. Capozziello, M. De Laurentis and E. N. Saridakis, Rept. Prog. Phys. 79, 106901 (2016), arXiv:1511.07586 [gr-qc]]. 
[23] R. C. Nunes, S. Pan, E. N. Saridakis and E. M. C. Abreu, JCAP 1701, 005 (2017), arXiv:1610.07518 [astroph.CO]].

[24] R. C. Nunes, S. Pan and E. N. Saridakis, JCAP 1608, 011 (2016), arXiv:1606.04359 [gr-qc]].

[25] J. Lu, M. Liu, Y. Wu, Y. Wang and W. Yang, Eur. Phys. J. C 76, 679 (2016), arXiv:1606.02987 [astro-ph.CO]].

[26] A. R. Cooray and D. Huterer, Astrophys. J. 513, L95 (1999), arXiv:astro-ph/9901097

[27] P. Astier, Phys. Lett. B 500, 8 (2001), arXiv:astro$\mathrm{ph} / 0008306$

[28] J. Weller and A. Albrecht, Phys. Rev. D 65, 103512 (2002), arXiv:astro-ph/0106079

[29] M. Chevallier and D. Polarski, Int. J. Mod. Phys. D 10, 213 (2001), arXiv:gr-qc/0009008

[30] E. V. Linder, 2003, Phys. Rev. Lett. 90, 091301 (2003), arXiv:astro-ph/0208512

[31] G. Efstathiou, Mon. Not. Roy. Astron. Soc. 310, 842 (1999), arXiv:astro-ph/9904356

[32] H. K. Jassal, J. S. Bagla and T. Padmanabhan, Phys. Rev. D 72, 103503 (2005), astro-ph/0506748.

[33] E. M. Barboza, Jr. and J. S. Alcaniz, Phys. Lett. B 666, 415 (2008), arXiv:0805.1713 [astro-ph]].

[34] E. V. Linder and D. Huterer, Phys. Rev. D 72, 043509 (2005), astro-ph/0505330.

[35] Q. Zhang, G. Yang, Q. Zou, X. Meng and K. Shen, Eur. Phys. J. C 75, no. 7, 300 (2015), arXiv:1502.01922 [grqc]].

[36] S. Nesseris and L. Perivolaropoulos, Phys. Rev. D 72, 123519 (2005), astro-ph/0511040.

[37] G. Pantazis, S. Nesseris and L. Perivolaropoulos, Phys. Rev. D 93, 103503 (2016), arXiv:1603.02164 [astroph.CO]].

[38] S. Nesseris and L. Perivolaropoulos, JCAP 0701, 018 (2007), astro-ph/0610092.

[39] S. Nesseris and L. Perivolaropoulos, JCAP 0702, 025 (2007), astro-ph/0612653.

[40] R. Adam, P. A. R. Ade, N. Aghanim, et al. [Planck Collaboration], Astron. Astrophys. 594, A1 (2016), arXiv:1502.01582.
[41] N. Aghanim, M. Arnaud, M. Ashdown, et al. [Planck Collaboration], Astron. Astrophys. 594, A11 (2016), arXiv:1507.02704.

[42] M. Betoule et al. [SDSS Collaboration], Astron. Astrophys. 568, A22 (2014), arXiv:1401.4064 [astro-ph.CO].

[43] F. Beutler et al., Mon. Not. Roy. Astron. Soc. 416, 3017 (2011).

[44] A. J. Ross, L. Samushia, C. Howlett, W. J. Percival, A. Burden and M. Manera, Mon. Not. Roy. Astron. Soc. 449, 835 (2015).

[45] H. Gil-Marín et al., Mon. Not. Roy. Astron. Soc. 460, 4210 (2016).

[46] C. Heymans et al., Mon. Not. Roy. Astron. Soc. 432, 2433 (2013), arXiv:1303.1808 [astro-ph.CO].

[47] M. Asgari, C. Heymans, C. Blake, J. Harnois-Deraps, P. Schneider and L. Van Waerbeke, Mon. Not. Roy. Astron. Soc. 464, 1676 (2017), arXiv:1601.00115 [astroph.CO].

[48] M. Moresco et al., JCAP 1605, 014 (2016), arXiv:1601.01701 [astro-ph.CO].

[49] A. G. Riess et al., Astrophys. J. 826, 56 (2016), arXiv:1604.01424 [astro-ph.CO].

[50] A. Lewis and S. Bridle, Phys. Rev. D 66, 103511 (2002), astro-ph/0205436.

[51] A. Lewis, Phys. Rev. D 87, 103529 (2013), arXiv:1304.4473 [astro-ph.CO]].

[52] A. Gelman and D. Rubin, Statistical Science, 7, 457 (1992).

[53] V. F. Mukhanov, H. A. Feldman and R. H. Brandenberger, Phys. Rept. 215, 203 (1992).

[54] C. P. Ma and E. Bertschinger, Astrophys. J. 455, 7 (1995), arXiv:astro-ph/9506072

[55] K. A. Malik and D. Wands, Phys. Rept. 475, 1 (2009), arXiv:0809.4944 [astro-ph].

[56] H. Akaike, IEEE Transactions of Automatic Control, 19, 716 (1974).

[57] N. Sugiura, Communications in Statistics A, Theory and Methods, 7, 13 (1978). 